\title{
Correction to: Research on multi-source POI data fusion based on ontology and clustering algorithms
}

\author{
$\mathrm{Li} \mathrm{Cai}^{1,2}$ (D) Longhao Zhu $^{1} \cdot$ Fang Jiang $^{1} \cdot$ Yihan Zhang $^{1} \cdot$ Jing He $^{1}$ \\ Published online: 19 October 2021 \\ (C) Springer Science+Business Media, LLC, part of Springer Nature 2021
}

\section{Correction to: Applied Intelligence. https://doi.org/10.1007/s10489-021-02561-6}

The original article unfortunately was published with errors. 1. Eq. (8) of Page 10: notation $|m|$ needs to be changed $m$.

2. Eq. (11) of Page 10: There is an error in Eqs. (11), the correct equation is as follows:

$F_{1}=2 \times \frac{\text { Precision } \times \text { Recall }}{\text { Precision }+ \text { Recall }}$

3. Left column of Page 11: in Table 7, and the results need to be changed in Tables 8.

4. Caption of Fig. 6 of Page 12: in Table 5 datasets need to be changed in Table 8 datasets.

5. Caption of Fig. 7 of Page 13: in Table 6 datasets need to be changed in Table 9 datasets.

The online version of the original article can be found at https://doi.org/ 10.1007/s10489-021-02561-6

Li Cai

caili@ynu.edu.cn

Longhao Zhu

longhaozhu@126.com

Fang Jiang

jiangfang918@mail.ynu.edu.cn

Yihan Zhang

yihanzhang@mail.ynu.edu.cn

Jing $\mathrm{He}$

hejing@ynu.edu.cn

School of Software, Yunnan University, Kunming 650091, China

2 School of Computer Science, Fudan University, Shanghai 200433, China
6. Caption of Fig. 8 of Page 14: in Table 8 data sets need to be changed in Table 11 datasets.

7. Caption of Fig. 9 of Page 14: in Table 8 data sets need to be changed in Table 11 datasets, and the font size of the caption in Fig. 9 is inconsistent with that in Fig. 8.

8. Fig. 10 of Page 15: all words data sets in caption need to be changed datasets, and the captions of Fig. 10(c) and Fig. 10(d) need to be center alignment.

9. According to Table 1, points $\mathrm{P} 2$ and $\mathrm{P} 3$ have very high name similarity: Table 1 changed to Table 6.

10. Caption of Fig. 5: evaluation indexes changed to evaluation metrics.

11. Caption of Fig. 6(a): evaluation indexes changed to evaluation metrics.

12. Captionof Fig. 9: evaluation indexes changed to evaluation metrics.

13. Captionsof Fig. 10(a)-(d): Fig. 10(a): running time of Table 5 datasets changed to running time inTable 8 datasets; Fig. 10(b): running time of Table 6 datasets changed to running time in Table 9 datasets; Fig.10(c): running time of Table 8 datasets changed to running time in Table 11 datasets; Fig. 10(d): under Table 8datasets changed to under Table 11 datasets.

The original article has been corrected.

Publisher's note Springer Nature remains neutral with regard to jurisdictional claims in published maps and institutional affiliations. 